\title{
Ciprofloxacin-induced Acute Generalized Exanthematous Pustulosis
}

Sahil Sheth*, Robert Chasse and Jeannette Crossan

University of Maryland Eastern Shore-School of Pharmacy, USA

Peninsula Regional Medical Center, USA

*Corresponding author: Sahil Sheth, University of Maryland Eastern Shore-School of Pharmacy, USA, Tel: 732-735-0593; E-mail: ssheth@umes.edu

Received date: Sep 18, 2018; Accepted date: Dec 10, 2018; Published date: Dec 14, 2018

Copyright: $@ 2018$ Sheth S, et al. This is an open-access article distributed under the terms of the Creative Commons Attribution License, which permits unrestricted use, distribution, and reproduction in any medium, provided the original author and source are credited.

\section{Abstract}

Acute Generalized Exanthematous Pustulosis (AGEP) is a cutaneous eruption characterized by sterile nonfollicular pustules located on erythematous skin. This case report describes a rare instance of ciprofloxacin-induced AGEP in a patient initially admitted to the hospital for treatment of acute diverticulitis.

Keywords: Exanthematous pustulosis; AGEP; SJS; Rash

\section{Introduction}

Severe Cutaneous Adverse Reactions (SCARs) are common side effects of antibiotics [1]. Acute Generalized Exanthematous Pustulosis (AGEP) is a cutaneous eruption characterized by sterile non-follicular pustules located on erythematous skin [2]. It is often associated with fever, malaise, and leukocytosis. Unlike Stevens-Johnson Syndrome (SJS) or Toxic Epidermal Necrolysis (TEN), it is rarely life-threatening with an average mortality rate of $2 \%$ [2]. We report a case of suspected ciprofloxacin-induced AGEP in a 59-year-old female.

\section{Case Report}

A 59-year-old female with a remote past medical history of nephrolithiasis, left-frontal hemorrhagic stroke, and necrotizing vasculitis was admitted on $8 / 2018$ to our hospital with acute diverticulitis with micro perforation. She was managed conservatively with intravenous (IV) fluids, NPO status, and IV antibiotics ciprofloxacin $400 \mathrm{mg}$ every 12 hours and metronidazole $500 \mathrm{mg}$ every 8 hours.

On day 3 of the hospital admission, a diffuse rash was noted on the patient's back and IV diphenhydramine therapy was initiated to provide symptom relief. At this time, the patient's family reported a remote history of rash from ciprofloxacin. The rash worsened over the next 2 days and due to increasing leukocytosis $(16.1 \times 109 \mathrm{~L})$ and reported allergy, antibiotics were changed to piperacillin-tazobactam 3.375 grams IV every 6 hours.

On day 6 , with continued worsening of rash and leukocytosis (24.4 $\times 109 \mathrm{~L}$ ) coupled with a lactate level of $2.8 \mathrm{mmol} / \mathrm{L}$, antibiotics were broadened further to aztreonam, vancomycin, and metronidazole (Table 1). Infectious disease consultant recommended continuing the current regimen empirically. Infectious workup included enterohemorrhagic assays and skin pustule smears, which remained negative. A dermatology consult was also obtained and a skin biopsy was performed. On day 9, the rash was now diffuse, edematous and erythematous with oral and vaginal mucosal involvement (Figures 1 and 2). A C-reactive protein level of $234.76 \mathrm{mg} / \mathrm{L}$ (reference range: $<7.48 \mathrm{mg} / \mathrm{L}$ ), lactate of $4.3 \mathrm{mmol} / \mathrm{L}$ (reference range: $0.4-2.0 \mathrm{mmol} / \mathrm{L}$ ), and intermittent episodes hypotension prompted a transfer to the intensive care unit for closer monitoring. Of note, no bullous lesions were noted and Nikolsky sign was negative. Differential diagnosis included Stevens-Johnson Syndrome, toxic epidermal necrolysis, pustular psoriasis, and staphylococcal scaled skin.

Pathology report of punch biopsy indicated several subcorneal pustules associated with moderate spongiosis and superficial dermal infiltrate without epidermal necrosis. The biopsy report combined with the patient's clinical picture led to a firm diagnosis of AGEP (Figures 3 and 4). All antibiotics were discontinued and triamcinolone cream $0.1 \%$ application and chlorhexidine bath were initiated. Rash and leukocytosis slowly improved with complete resolution of rash on day 13 of hospitalization. The patient was discharged on hospital day 17 with the recommendation of the topical application of white petrolatum as needed daily. Liver and renal function tests remained normal throughout hospitalization. Of note, patch testing was not performed.

\begin{tabular}{|l|l|}
\hline Age & 59 \\
\hline Sex & Female \\
\hline Drug allergy & Ciprofloxacin \\
\hline Previous drug reaction & Rash \\
\hline Interval between drug intake and rash & 3 days \\
\hline Interval between drug intake and pustules & 9 days \\
\hline Duration of rash & 13 days \\
\hline Fever>38.75 ${ }^{\circ} \mathrm{C}$ & Yes \\
\hline Leukocytes & 34500 \\
\hline Neutrophils & $94 \%$ \\
\hline C-reactive protein & 234.76 mg/L \\
\hline Lactate & 4.3 mmol/L \\
\hline Skin biopsy results & $\begin{array}{l}\text { with moderate spongiosis and } \\
\text { superficial dermal infiltrate }\end{array}$ \\
\hline
\end{tabular}


Resolution of rash after drug withdrawal

10 days

Table 1: Relevant patient characteristics and laboratory results.

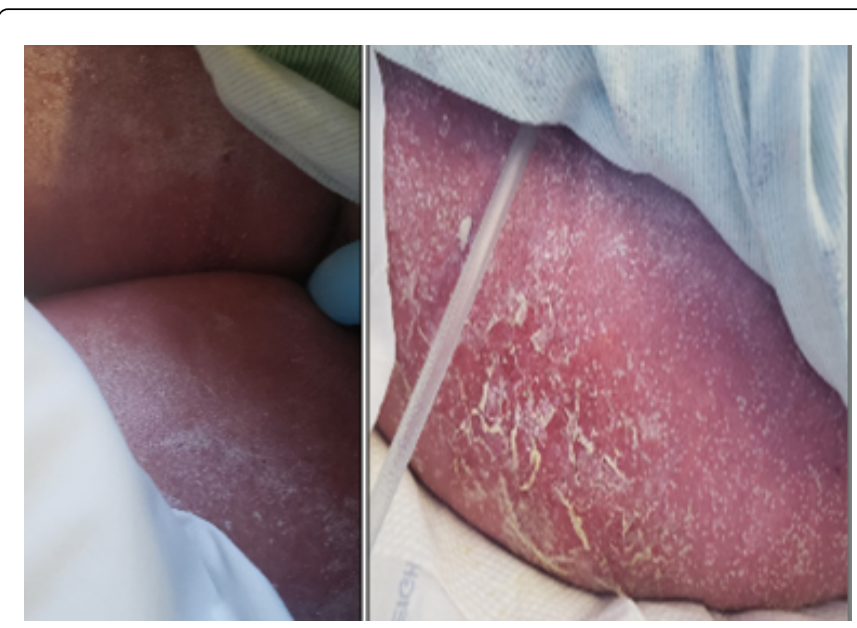

Figure 1: Diffuse rash seen on inner thighs (left) and left hip (right) on Day 5 of hospitalization.

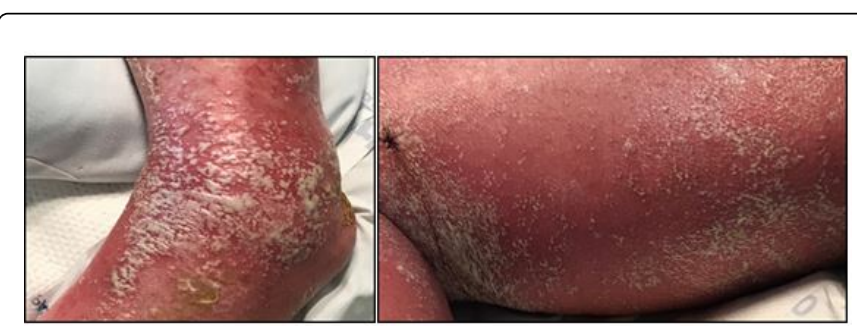

Figure 2: Significant pustular erythematous rash seen on left foot (left) and left hip (right) on day 9 of hospitalization.

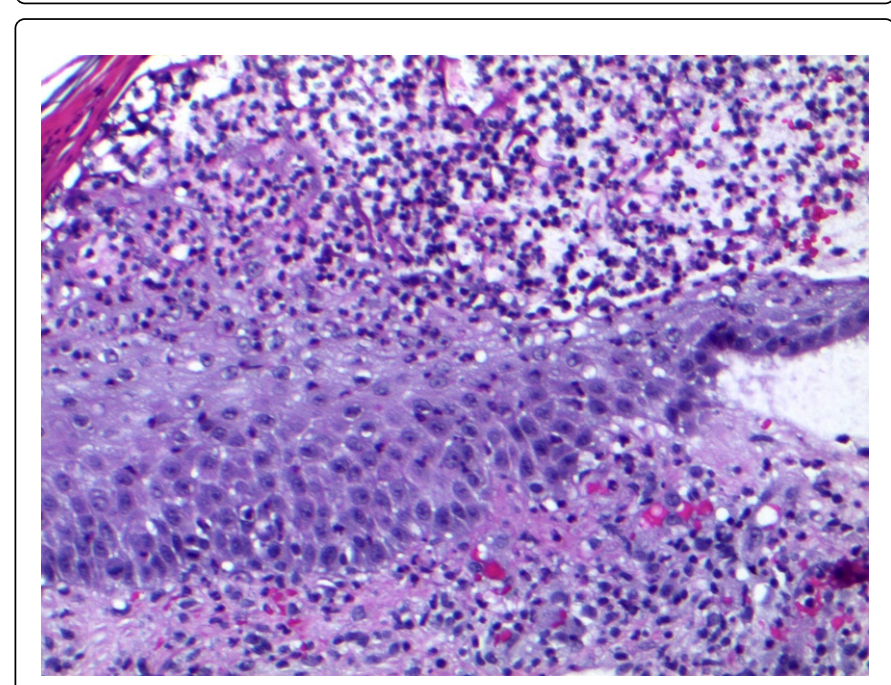

Figure 3: High power view of a subcorneal pustule showing numerous neutrophils within a pustule, mild/moderate spongiosis and a mild superficial dermal lymphocytic infiltrate.

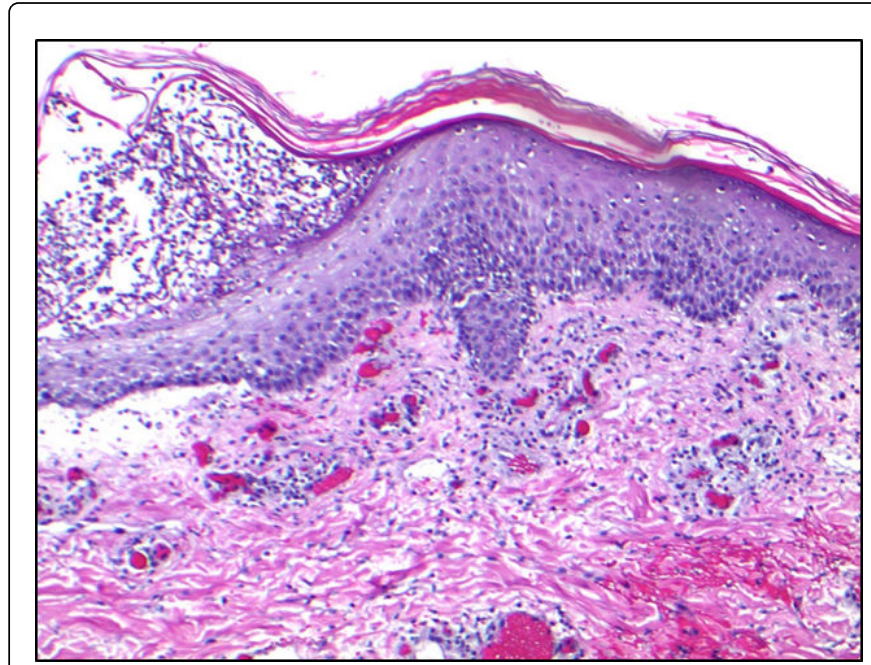

Figure 4: Low power view demonstrating prominent spongiotic subcorneal pustule. Acanthosis is not prominent. A mild perivascular lymphocytic infiltrate is present in the superficial dermis.

\section{Discussion}

This case demonstrates a rare instance of ciprofloxacin-induced AGEP. While AGEP is known to be self-limiting after drug discontinuation, severe cases have been reported in elderly and immunocompromised patients [1]. Commonly, antibiotics implicating in causing AGEP are penicillins, cephalosporins, and fluoroquinolones [1]. The average length before AGEP eruption is 1-11 days, with antibiotics causing an earlier symptom-onset [3]. Faster courses have been observed when patients are re-challenged with an offending agent, regardless of the severity of the initial reaction [1]. In this case, the patient was re-exposed to ciprofloxacin and symptom onset was approximately 1.5 days. Typical clinical features of this case included diffuse, progressive edematous and erythematous rash and a consistent histological pattern accompanied by fevers, neutrophilia, and leukocytosis [2]. Atypical symptoms included involvement of mouth and vaginal mucosa, only seen in $20 \%$ of AGEP cases [1]. AGEP validation score derived through the EuroSCAR criteria was 9, signifying "definite" AGEP based on morphology, clinical course, and histology (range for definite AGEP: 8-12) [4].

To our knowledge, this is the fourth reported case of ciprofloxacininduced AGEP, with only one, confirmed via patch testing [5]. We cannot fully rule out metronidazole-induced AGEP.6 However, due to patient's previously reported allergy to ciprofloxacin, acuteness of symptom-onset with re-exposure and lack of metronidazole-induced AGEP case reports, it is reasonable to attribute this case to ciprofloxacin. Patch testing is a useful and noninvasive way to confirm the culprit agent but is characterized by sensitivity of only $60 \%$ [6]. After reviewing this case, antibiotic stewardship, a thorough review of allergies, and early recognition of cutaneous reactions are essential for appropriate clinical diagnosis and improved patient care. 
Citation: Sheth S, Chasse R, Crossan J (2018) Ciprofloxacin-induced Acute Generalized Exanthematous Pustulosis. Dermatol Case Rep 3: 144.

Page 3 of 3

\section{References}

1. Tajmir-riahi A, Wörl P, Harrer T, et al. (2017) Life-threatening atypical case of acute generalized exanthematous pustulosis. Int Arch Allergy Immunol 174: 108-111.

2. Kostopoulos TC, Krishna SM, Brinster NK, et al. (2015) Acute generalized exanthematous pustulosis: atypical presentations and outcomes. J Eur Acad Dermatol Venereol 29: 209-214.

3. Sidoroff A, Dunant A, Viboud C, et al. (2007) Risk factors for acute generalized exanthematous pustulosis (AGEP)-results of a multinational case-control study (EuroSCAR). Br J Dermatol 157: 989-996.
4. Sidoroff A, Halevy S, Bavinck JN, et al. (2001) Acute generalized exanthematous pustulosis (AGEP)-a clinical reaction pattern. J Cutan Pathol 28: 113-119.

5. Hausermann P, Scherer K, Weber M, et al. (2005) Ciprofloxacin-induced acute generalized exanthematous pustulosis mimicking bullous drug eruption confirmed by a positive patch test. Dermatology (Basel) 211: 277-280.

6. Foti C, Romita P, Zanframundo G, et al. (2017) Ciprofloxacin induced acute generalized exanthematous pustulosis. Indian J Pharmacol 49: $119-120$ 